\title{
Is near-surface ice the driver of dust activity on $67 \mathrm{P} /$ Churyumov-Gerasimenko
}

\author{
Yu. V. Skorov ${ }^{1}$, L. Rezac ${ }^{1}$, P. Hartogh ${ }^{1}$, and H. U. Keller ${ }^{2}$ \\ ${ }^{1}$ Max-Planck-Institut für Sonnensystemforschung, Justus-von-Liebig-Weg 3, 37077 Göttingen, Germany \\ e-mail: skorov@gmail.com \\ 2 Institute for Geophysics and Extraterrestrial Physics, TU Braunschweig, 38106 Braunschweig, Germany \\ Received 2 November 2016 / Accepted 30 December 2016
}

\begin{abstract}
Context. Mainly for historical reasons, nearly all of the current thermophysical models of dust activity rely on the poorly justified assumption of cohesionless dust lifted by a gas drag force against the weak nucleus gravity. The interpretation of Rosetta data and our understanding of comet activity is particularly sensitive to this assumption.

Aims. We investigate the role that cohesion forces among the dust grains play in the evolution of temperature and pressure at the ice-dust interface and the resulting dust activity (lifting).

Methods. We used a 1D thermophysical numerical model that provides a realistic description of cohesion forces among dust aggregates. Several conditions of solar illumination on the nucleus are investigated for the $\mathrm{H}_{2} \mathrm{O}, \mathrm{CO}$, and $\mathrm{CO}_{2}$ ices below the dust layer. We examine a wide range of dust grain sizes.

Results. The simulations confirm an increase in temperature and pressure at the ice boundary between the two model layers with respect to exposed pure ice. Furthermore, we show that a non-monotonic behavior of temperature and pressure versus layer thickness is expected at the ice-dust interface for fine aggregates (of sizes $\leq 30 \mu \mathrm{m}$ ), but not for the larger grains. The ratio of vapor pressure to the physically determined tensile strength for various agglomerate sizes and layer thicknesses provides further evidence that the gas drag is not sufficient to remove dust grains of sizes $<1 \mathrm{~mm}$, which is a result of taking cohesion forces among the particles into account.

Conclusions. In the framework of the presented model, which can be considered common in terms of assumptions and physical parameters in the cometary community, the dust removal by a gas drag force is not a plausible physical mechanism. The sublimation of not only water ice, but also of super-volatile ice (i.e., $\mathrm{CO}$ ) is unable to remove dust grains for illumination conditions corresponding to 1.3 AU. A way out of this impasse requires revision of the most common model assumption employed by the cometary community.
\end{abstract}

Key words. comets: general - comets: individual: comet 67P/Churyumov-Gerasimenko - methods: numerical

\section{Introduction}

Dust lifted from a comet nucleus provides enough surface to scatter sunlight, which is the defining characteristic of these small bodies that have attracted our attention for millennia. Although the discussion of dust ejection from cometary nuclei was the focus of many researches starting from the pioneering article of Whipple (Whipple 1950), even today our understanding of this process is far from complete in many respects. The results obtained during the Rosetta mission brought new puzzles and questions.

The dust activity on 67P/Churyumov-Gerasimenko (67P) has been detected at surprisingly large heliocentric distances ( 4.3 AU) (Tubiana et al. 2015; Moreno et al. 2016). Schulz et al. (2015) reported about the grain collection commenced by COSIMA at a heliocentric distance of 3.57 AU. Rotundi et al. (2015) described the dust observations by GIADA and OSIRIS at distances from 3.7 to 3.4 AU. Later the outstanding results related to the study of comet dust were published by other teams that had instruments on board of ESA's Rosetta spacecraft. We list below the main results that are connected with the topic of this paper. From the OSIRIS instrument results we learned that 1) dust activity is observed everywhere, it is not localized only in jets and/or active regions (hence dust is most likely released from the surface); 2) light scattering from the dust particles is clearly visible in the closest vicinity of the nucleus; and 3) particle sizes vary from micrometers up to meters. A large number of dust grains have been investigated in situ with COSIMA (Langevin et al. 2016), GIADA (Della Corte et al. 2015), and MIDAS (Bentley et al. 2016), clearly indicating that the majority of these particles have a high porosity ("fluffy" aggregated grains), most likely of hierarchical structure (i.e., larger aggregates consist of small fluffy aggregates). Finally, there is also strong evidence that the material of cometary nuclei possesses non-zero cohesion (Groussin et al. 2015; Hilchenbach et al. 2016; Spohn et al. 2015). Therefore, results from multiple instruments available so far point into the direction of continuous cometary activity, where small fluffy cohesive dust particles (also of sizes $<1 \mathrm{~mm}$ ) are released from the surface layer of the nucleus. Then, the relevant open questions we would like to address here are connected with the mechanism of this dust release, and whether the current models can explain this phenomenon.

A typical scenario considered in the majority of models treats the dust lifting as an ejection directly off of the comet nucleus surface. In all cases, the dust removal requires the sum of lifting forces to be greater than the "resisting" forces. A variety of physical processes has been investigated as the potential drivers of the cometary dust release. Micrometeorite bombardment of the surface (Chapman 2004), electrostatic charging of the nucleus 
(Mendis et al. 1981; Nordheim et al. 2015), and the mechanism of ion-stimulated erosion due to an interaction of cometary nuclei with the solar wind (Kalinichenko et al. 1996) have been examined. However, the lifting force that arises as a result of nearsurface ice sublimation is considered as the most common lifting force since the article of Whipple. On the other hand, only the nucleus gravity is typically considered as the opposing force. It is implicitly assumed in these models that the cometary material is non-cohesive, and only a balance of forces acting on a detached dust grain is evaluated. Some prominent examples of such investigations can be found in Rickman et al. (1990), Huebner et al. (2006).

In the seminal paper of Kuehrt \& Keller (1994) it was clearly recognized that the non-volatile material of the cometary nucleus is most likely cohesive. Assuming the weak van der Waals forces among solid spherical grains, the authors showed that the (cohesive) strength of the homogeneous porous surface dry dust layer is about $0.1 \mathrm{kPa}$ and $100 \mathrm{kPa}$ for millimeter and micron size particles, respectively. This led to the conclusion that the lifting force of sublimating ice is completely insufficient to remove particles from the porous dust surface layer. The authors also pointed out that the dry porous crust coating the sublimating icy medium may lead to an increase in vapor pressure on the icy boundary (the so-called "cooking effect", Skorov et al. 1999). Nevertheless, even with this model improvement the cohesive strength of refractory material generally far exceeds the expected vapor pressure of sublimating water ice under the surface dust crust. Because the authors did not offer a solution to the problem, the crucial role of cohesion for cometary dust ejection has been largely ignored so far by the cometary community. Models excluding a cohesion are widely used until now, including the most recent literature interpreting the Rosetta measurements (Nordheim et al. 2015; Marschall et al. 2016; Lin et al. 2016; Kramer \& Noack 2016).

Although the problem of the release of dust from the surface porous layer was not solved in Kuehrt \& Keller (1994), the authors have identified two basic ideas for its solution: either the lifting force of sublimating ice must be substantially increased, or the tensile strength (cohesion) of dusty media has to be dramatically reduced. Because the lifting force of the expanding gas is directly proportional to its density, it is natural to evaluate sublimation of super volatile ices (such as $\mathrm{CO}$ ).

The second idea was investigated until Skorov \& Blum (2012) suggested an original model, where the comet material features a low cohesion strength due to its micro-structure. They developed a thermophysical model for a comet based upon the assumption that the near-surface layer of cometary nuclei is formed by an ensemble of dust and ice porous aggregates (i.e., its porosity has hierarchical character). Under this condition, the tensile strength of the ice-free outer layers was evaluated using the dust-aggregate collision and adhesion model of Weidling et al. (2012). Based on available laboratory data, the authors derived the temperature and pressure at the dust-ice interface for pure water and pure carbon dioxide ice. The model predicted possible dust activity for near-surface $\mathrm{CO}_{2}$ ice for heliocentric distances of $\lesssim 3 \mathrm{AU}$, whereas sublimation of nearsurface $\mathrm{H}_{2} \mathrm{O}$ ice could not provide the dust emission. We note that the authors made several model simplifications (for example, the comet gravity was ignored, the effective conductivity was considered to be constant, and the heat flux to the interior was linearly approximated).

The next model where the dust lifting conditions for a hierarchic porous cohesive dust crust were determined from a physically meaningful balance of forces was presented in Gundlach et al. (2015). This model included the force due to gravity of the cometary nucleus, as well as the strength of the porous non-volatile material, and the vapor pressure of underlying sublimating ice in a consistent manner. Because the tensile strength of the porous aggregates dramatically decreases with increasing aggregate size, the model could explain the large grains (ranging from $2 \mathrm{~cm}$ to $1 \mathrm{~m}$ in radius) in the inner coma of comet 67P observed by the OSIRIS camera at heliocentric distances between 3.4 AU and 3.7 AU (Davidsson et al. 2015; Rotundi et al. 2015). It was also shown that it is possible for super-volatile ice (CO) sublimation to detach aggregates that are smaller by approximately an order of magnitude in comparison with pure water ice, but under the assumption that $\mathrm{CO}$ ice can exists in near surface layer. Nevertheless, the gas-driven dust activity could not explain particles smaller than $1 \mathrm{~mm}$ in the coma because of the high tensile strength required to detach these particles from the surface. It should be noted that the work still relied on serious simplifications: heat conductivity and the gas diffusion resistance of the near-surface porous layer were neglected, similar as in Skorov \& Blum (2012).

In this paper we present a microphysical model taking all general physical processes and available theoretical as well as experimental results into consideration. The aim of this research is to investigate the heat and mass transport in a porous hierarchic near-surface layer in order to estimate the acting forces and conditions for possible removal of small dust grains, typically detected by the Rosetta instruments. The paper is structured as follows. In Sect. 2 we describe a physical two-layer model and discuss basic model assumptions, while the main thermophysical results are presented in Sect. 3, the dust removal conditions are discussed in Sect. 4, and in Sect. 5 we summarize the main results and outline future steps in applying and validating the current model.

\section{Thermophysical modeling}

The cometary dust in the model is generally treated as porous aggregates. The surface is covered by a dark thin ice-free layer that forms when the ice is sublimated. After losing its ice content, the dry dust layer slightly collapses, but its morphology stays similar to the initial morphology of the ice-dust mixture. The pristine ice-dust medium is located below this refractory layer. The cohesive forces between dust particles are typically smaller than for ice and dust connections, therefore it seems physically reasonable that dust is released from a porous dry layer on the surface (Skorov \& Blum 2012; Gundlach et al. 2015). Ejection of particles larger than the thickness of the dust layer is not considered in the model.

To evaluate the gas-lifting force, we focus primarily on vapor pressure under a dry dust layer overlaying the sublimating icedust mixture. The main equations were presented in the pioneering work of Fanale \& Salvail (1984), and our new model is described in detail in Skorov \& Blum (2012), Keller et al. (2015). This is a 1D heterogeneous two-layer model of the nucleus, which means that the ice-free dust layer is treated explicitly and its thickness is a free model parameter. The ice sublimation is included in the model. Because of an ice-free porous dust layer, the resulting gas production and the outward impulse of the gas flow depends on the diffusive permeability of the layer. The nucleus surface erosion is not included in this model because we assume that the characteristic time of the gas and the heat diffusion are much shorter than variations due to solar irradiation. The boundary conditions on the surface and at the lower boundary 
of the dust layer express the energy conservation:

$$
\left(1-A_{v}\right) I_{v} R_{\mathrm{H}}^{-2}=\epsilon \sigma T_{\mathrm{s}}^{4}+\lambda_{\mathrm{dust}}(T) \frac{\mathrm{d} T}{\mathrm{~d} x}
$$

$\lambda_{\text {dust }}(T) \frac{\mathrm{d} T}{\mathrm{~d} x}=\Psi\left(R_{\mathrm{A}}, L\right) Z(T) H(T)+\lambda_{\text {core }}(T) \frac{\mathrm{d} T}{\mathrm{~d} x} ;$

here $I_{v}$ is the solar irradiation, $R_{\mathrm{H}}$ is heliocentric distance, $A_{v}$ is the albedo, $\epsilon$ is the emissivity, $\sigma$ is the Stefan-Boltzmann constant, $T_{\mathrm{s}}$ is the surface temperature, $Z$ is the sublimation rate, and $H$ is the latent heat of sublimation. The parameters $\lambda_{\text {dust }}(T)$ and $\lambda_{\text {core }}(T)$ denote the effective heat conductivity of the dust and core layer, respectively.

The sublimation rate is given by the Hertz-Knudsen formula, $Z(T)=P(T) /\left(0.5 \pi v_{\mathrm{th}}\right)$, where the water saturation vapor pressure is $P(T)=3.56 \times 10^{12} \times \exp (-6141.667 / T) \mathrm{kg} \mathrm{m}^{-1} \mathrm{~s}^{-2}$ (Fanale \& Salvail 1984) and the thermal velocity $v_{\mathrm{th}}(T)=$ $\sqrt{8 R T / \pi \mu} \mathrm{m} \mathrm{s}^{-1}$, and $\mu$ is the molar mass of water. The permeability of the ice-free porous dust layer, $\Psi\left(R_{\mathrm{A}}, L\right)$, is a function of the agglomerate radius $R_{\mathrm{A}}$ and the thickness of the dust layer $L$. For the collisionless gas the mass flow through the pore volume is calculated by a modified Clausing formula (Skorov et al. 2011). For the results presented in Sect. 4 the experimental dependence (Gundlach et al. 2011) is also used. The model explicitly treats the solid and radiative part of the effective conductivity, which leads to a temperature dependence of the effective conductivity (Gundlach \& Blum 2012). We assume that the solid conductivity of the dust crust and the ice-dust mixture are the same and that the material porosity is constant everywhere, similar as in (Skorov \& Blum 2012; Keller et al. 2015; Skorov et al. 2016).

\section{Vapor pressure below the dust layer}

Using the model described above, we first examine the behavior of the vapor pressure at the interface between the ice-dust mixture and the dry dust layer as a function of thermophysical characteristics. Since the diffusive permeability and effective thermal conductivity of the dust layer are determined coherently by its micro-physical variables, the model parameter space is reduced to only two: the agglomerate size (radius $R_{\mathrm{A}}$ ), and the layer thickness $L$. The agglomerate size changes from $1 \mu \mathrm{m}$ to $1 \mathrm{~mm}$ (this size limitation is explained in Sect. 5), and the dimensionless layer thickness $\left(L / R_{\mathrm{A}}\right)$ varies from 2 to $>100$ for fine agglomerates. The effective conductivity controls the heat flux into the icy surface, and it depends on the agglomerate size, such that the solid thermal conductivity decreases with increasing size of dust grains, while the radiative heat conductivity increases. First we demonstrate the effects of incoming radiative flux $\left(I_{v}\right)$ to simulate the variation in heliocentric distance and/or local orientation of the nucleus surface. Following (Gundlach et al. 2015) the $\mathrm{H}_{2} \mathrm{O}, \mathrm{CO}_{2}$, and $\mathrm{CO}$ ice sublimation is investigated as representing different volatility. In all cases a mixture of the dust and one component ice is modeled. The main results of our simulations are shown in Figs. 1, 2.

\section{1. $\mathrm{H}_{2} \mathrm{O}$ ice}

The behavior of a mixture of water ice and dust covered by an ice-free porous layer at the perihelion distance $R_{\mathrm{H}}=1.243 \mathrm{AU}$ is shown in Fig. 1, first row, for water ice. The dependence of the surface temperature of the dust layer $T_{\mathrm{S}}$ on the $L / R_{\mathrm{A}}$ is shown in the left panel. For all cases when the layer thickness is much greater than the agglomerate size $\left(L \gg R_{\mathrm{A}}\right), T_{\mathrm{s}}$ approaches the equilibrium temperature of a blackbody (horizontal solid line), as expected. However, for a thin dust layer, a sublimating (and hence cool) icy surface becomes noticeable. This shows that the dimensionless thickness plays a crucial role. A dust layer of $2 \mathrm{~mm}$ thickness constructed from millimeter grains is about $60 \mathrm{~K}$ colder than a layer of the same thickness constructed from thousand-micron-sized grains. It therefore follows that the surface temperature does not indicate the layer thickness. At the same time, considerable deviation of a surface temperature from the corresponding blackbody temperature can be treated as an indication of a sublimating ice or small dimensionless thickness of the covering dust layer.

The behavior of the ice (interface) temperature $T_{\mathrm{i}}$ is even more interesting (middle panel). For small agglomerates $\left(R_{\mathrm{A}} \leq\right.$ $20-30 \mu \mathrm{m}$ ) the temperature of ice overlaid by a dust layer is slightly but clearly higher than the temperature of bare ice irradiated by the same flux, which shows the so-called "cooking effect". For a certain value of the layer thickness this temperature increment reaches a maximum, but then begins to decrease as the layer thickness increases further. For larger grains $\left(R_{\mathrm{A}}>30 \mu \mathrm{m}\right)$ the temperature decreases monotonically with increasing layer thickness. Therefore, the vapor pressure below the cover layer of dust does not increase. In the numerical experiment with the largest grains $\left(R_{\mathrm{A}} \approx 1 \mathrm{~mm}\right)$ the layer thickness is so great that the ice surface temperature is lower than the bare ice that absorbs the equal amount of energy (dashed line).

Because the saturation vapor pressure is a monotonic function of ice temperature, the temperature response is directly transferred to the pressure, as shown in right panel. As expected, the layer thickness at which this maximum pressure is achieved is also a function of agglomerate size. For example, in the case of relatively fine grains $\left(R_{\mathrm{A}} \leq 30 \mu \mathrm{m}\right)$, a pressure maximum exists, and its absolute value is a sensitive function of the agglomerate size. At $1 \mathrm{AU}$ the maximum saturation water vapor pressure below a porous dust layer built from micron grains is about $10 \mathrm{~Pa}$, that is, 30 times higher than the saturation pressure of pure water ice. Two different types of curves (for fine and coarse particles) suggest two qualitative scenarios of a layer evolution. When only the coarse grains are present, the layer growth leads to a monotonic decrease of the icy surface temperature, therefore to a pressure decrease with increasing thickness. When this layer starts to form, it grows monotonically, and the gas activity will be quenched quickly (because the layer permeability and hence gas production decreases in inverse proportion to the thickness even in the case of a fixed interface temperature). For very fine grains, the layer grows for some time, and so does the pressure, although it may not reach a clear maximum value. When the lifting force exceeds the material tensile strength during the pressure buildup, the layer will be destroyed (partially or completely). This scenario was first considered in general by Skorov \& Blum (2012).

\section{2. $\mathrm{CO}_{2}$ ice}

The behavior of a mixture of carbon dioxide ice covered by an ice-free porous dust layer at $1 \mathrm{AU}$ is shown in the middle row of Fig. 1. Although in the comet literature this ice is often referred to as super volatile, its thermodynamic characteristics are somewhat similar those of water ice. For the rate of sublimation and the gas lifting force, it is enough to compare the energy of sublimation per molecule in the zero approximation. The $\mathrm{H}_{2} \mathrm{O}$ ice latent heat of sublimation is $\Lambda=2.6 \times 10^{6} \mathrm{~J} \mathrm{~kg}^{-1}$ (Orosei et al. 1995) and for $\mathrm{CO}_{2} \Lambda=5.7 \times 10^{5} \mathrm{~J} \mathrm{~kg}^{-1}$ (Mavko et al. 2009), 
A\&A 600, A142 (2017)
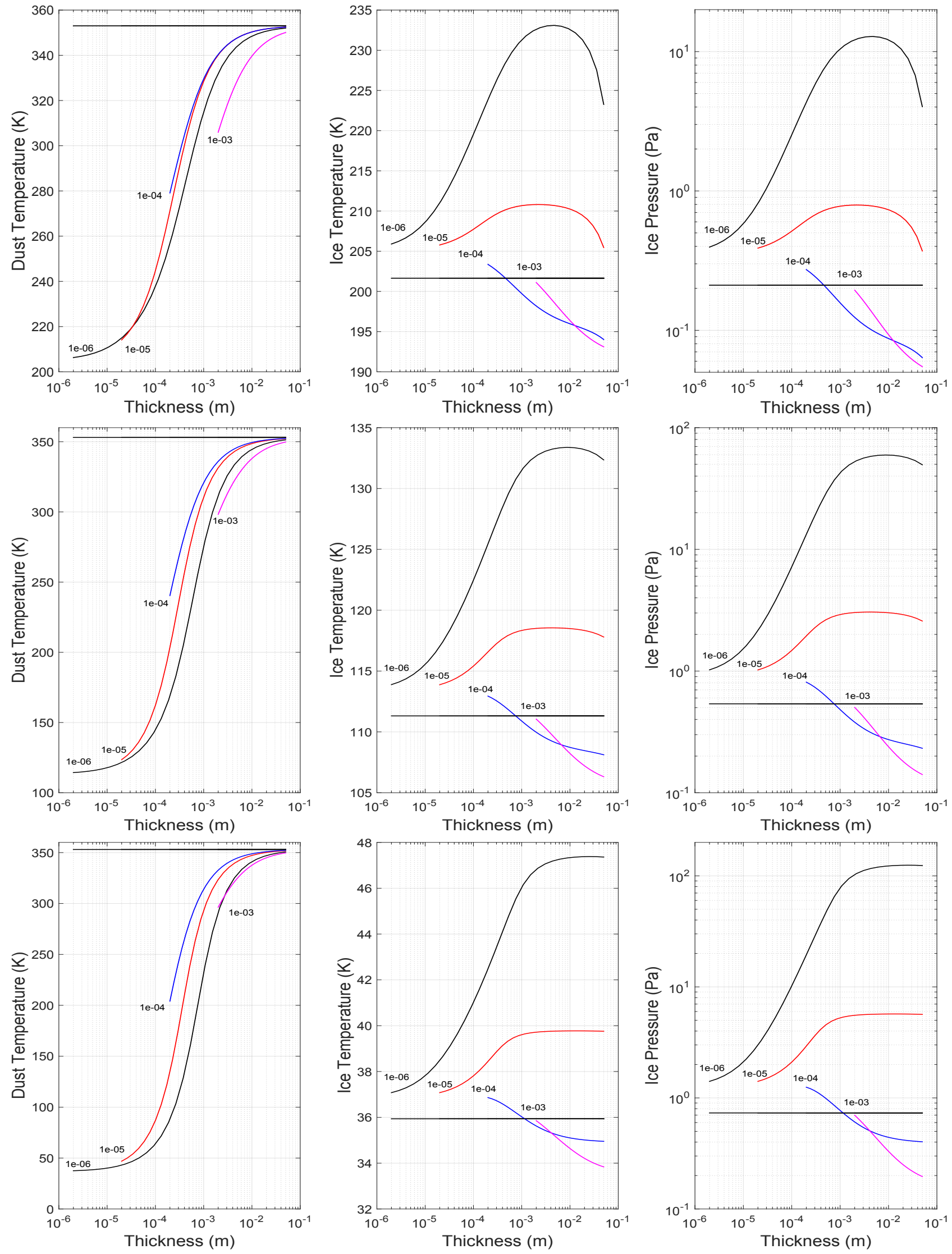

Fig. 1. Surface temperature (left column), the interface temperature (middle column), and the vapor pressure (right column) as a function of the layer thickness at perihelion distance. Results are plotted for water (top row), carbon dioxide (middle row), and carbon monoxide (bottom row) ices. Aggregate radii are shown as labels on the curves. The horizontal lines indicate the blackbody temperature (left column), the bare ice temperature (middle), and the corresponding saturation pressure (right). 
Skorov et al.: Dust release with cohesion
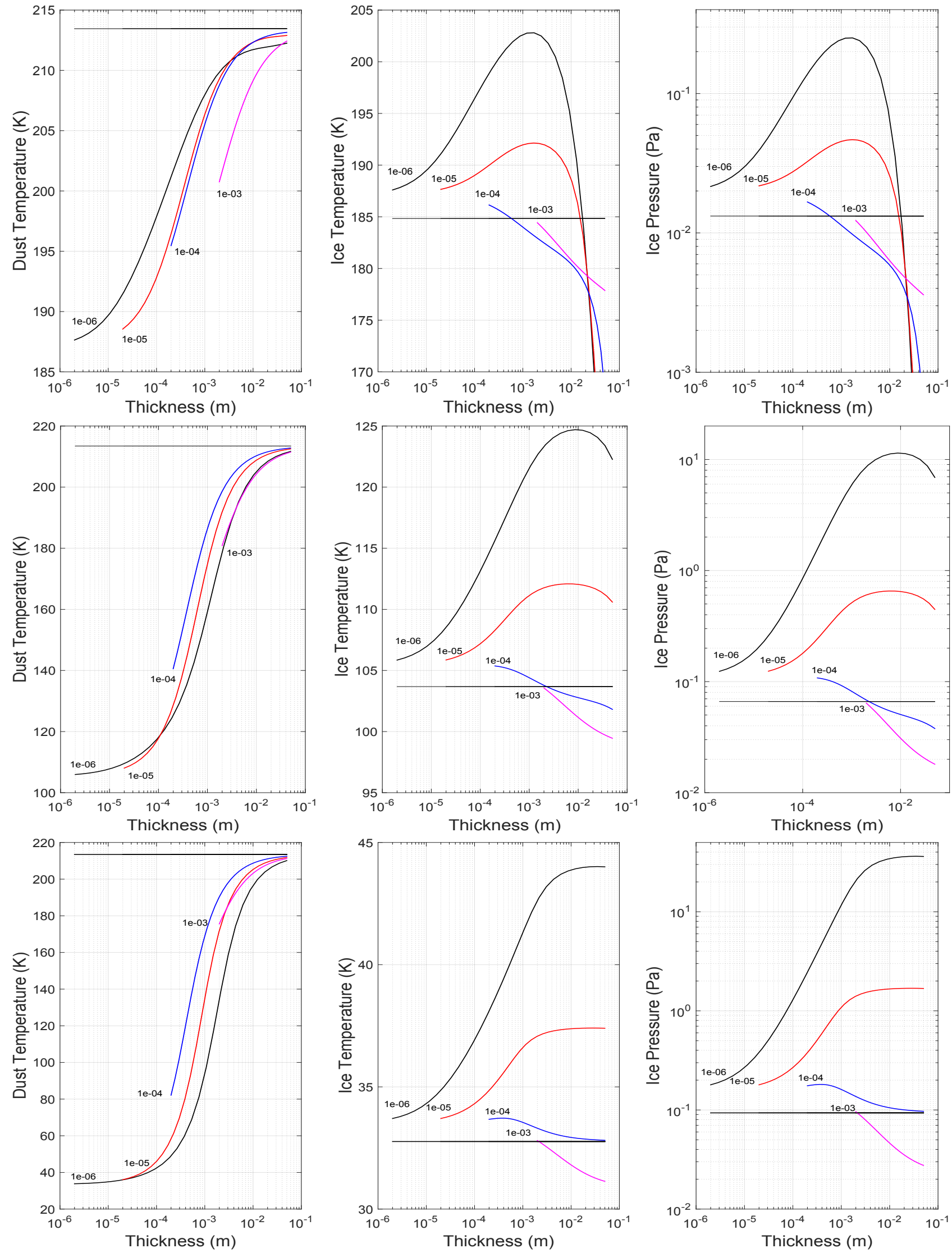

Fig. 2. Surface temperature (left column), the interface temperature (middle column), and the vapor pressure (right column) as a function of the layer thickness at the heliocentric distance $R_{\mathrm{H}}=3.4 \mathrm{AU}$. Results are plotted for water (top row), carbon dioxide (middle row), and carbon monoxide (bottom row) ices. Aggregate radii are shown on the curves. The horizontal lines indicate the blackbody temperature (left column), the bare ice temperature (middle), and the corresponding saturation pressure (right). 
which means a ratio of only 4.6. Our systematic calculations have shown that this is not enough to qualitatively change the character of the solution presented for $\mathrm{H}_{2} \mathrm{O}$ (see first row in Fig. 1). All the specific features of temperature and pressure versus aggregate size remain the same, although the effects are amplified. At $1 \mathrm{AU}$ the maximum pressure for one micron grains is about several hundred times higher than the saturation pressure of the bare $\mathrm{CO}_{2}$ ice and is about $70 \mathrm{~Pa}$.

\section{3. $\mathrm{CO}$ ice}

We finally consider a model where the mixture of $\mathrm{CO}$ ice and dust is present in the vicinity of the surface, as in previous cases (bottom row of Fig. 1). Although this assumption is not completely physically sound, these results give us an upper bound for the vapor saturation pressure. The equilibrium temperature of pure CO ice is so low that heat flux to the interior is also very low. This means that pressure at the interface is a weaker function of the layer thickness. However, there are still two types of solution, one for fine grain sizes $\left(R_{\mathrm{A}}<0.1 \mathrm{~mm}\right)$, and another for a coarse grain size (bottom row right panel). In the case of small dust particles the vapor pressure grows monotonically, resulting in a horizontal asymptote, whereas it decreases for the larger grains. In addition, since the latent heat of $\mathrm{CO}$ ice sublimation is nearly twice as low as the sublimation latent heat of $\mathrm{CO}_{2}$ ice, the absolute maximum values of pressure are higher than the $\mathrm{CO}_{2}$ vapor pressure (for the same grain sizes).

Similar numerical experiments were performed for a decreased radiation intensity (i.e., illumination conditions at $R_{\mathrm{H}}=$ $3.4 \mathrm{AU})$. The results do not change qualitatively for any of the three ices under consideration, and all the effects discussed above still hold (Fig. 2). However, because of the lower energy input, the radiation heat conductivity has decreased by about five times for millimeter particles. The difference between the blackbody temperature and the bare icy surface temperature is smaller at large heliocentric distance for all considered ices. As a result, the variability of the three diagnostic quantities $\left(T_{\mathrm{i}}, T_{\mathrm{s}}\right.$, pressure $)$ is also lower.

\section{Tensile strength and lifting force}

To investigate the specific conditions of dust removal by the gas lifting force, we have to evaluate the tensile strength of a model porous bed built from the dust aggregates (hierarchic porous layer). We follow the model of Skorov \& Blum (2012). The tensile strength of the dust aggregate layer is a function of its porosity and the size of aggregates,

$$
Y=Y_{0} \phi s^{-2 / 3} \text {. }
$$

$Y_{0}=1.6 \mathrm{~Pa}$ is a constant, $\phi$ is the volume-filling factor of the aggregate layer, and $s$ is the dust aggregate radius measured in millimeters. Brisset et al. (2016) experimentally showed that Eq. (3) is quantitatively correct (see their Fig. 14). It must be stressed that the tensile strength of the porous layer diminishes with increasing particle size. This relationship is obtained both theoretically (Skorov \& Blum 2012) and experimentally. Thus, a lifting force required to remove the dust layer (or destroy it) decreases rapidly with increasing aggregate radius. For the ice-free hierarchic porous layer, the tensile strength of millimeter-sized agglomerates is below one Pascal.

A typical formulation of dust removal requires that the gas pressure below a porous layer should exceed the cohesion strength of the layer. It is also important to note that the dust layer break (destruction) always occurs in the place of the weakest cohesion and that the vapor pressure above sublimating ice cannot exceed the corresponding saturated vapor pressure. Therefore we can immediately use the estimated $P_{\mathrm{s}}$ in the ratio to the tensile strength as a function of agglomerate size and layer thickness, as shown in Fig. 3.

If the ratio is greater than one, we can expect that the dust layer will be entirely removed. In this simplification, we consider only the pressure at the interface of ice and dust layer. It is clear that for larger particles (when the pressure decreases monotonically with increasing layer thickness) this ratio is an overestimation. However, even for this optimistic evaluation the conclusion is not promising. At 3.4 AU even the most volatile ice (CO) cannot remove dust grains smaller than $\sim 1 \mathrm{~mm}$ (panel b). For conditions at 1.243 AU the ratio is greater than one for the micron-sized agglomerates and also for agglomerates larger than $1 \mathrm{~mm}$ for the sublimating $\mathrm{CO}$ and $\mathrm{CO}_{2}$ ices. It is important to note that for the micron-sized grains the ratio exceeds 1 only when the layer thickness is several hundred microns, that is, when the layer is entirely removed. The pieces of the destroyed layer can have a variety of sizes (smaller or even slightly larger than the layer thickness). However, using micronsized agglomerate, we cannot instantly produce micron-detached grains. They are probably produced through secondary fragmentation (Keller et al. 1990; Combi 1994). In the case of large agglomerates $(>1 \mathrm{~mm})$, the sublimation process can only remove individual agglomerates and not pieces of layer, since the ratio is highest for the thinnest layer. The sublimation pressure of less volatile ices $\left(\mathrm{CO}_{2}\right.$ and $\left.\mathrm{H}_{2} \mathrm{O}\right)$ cannot remove the layer or lift particles from the surface at all.

In the previous discussion, the vapor pressure above the sublimating ice was considered as the lifting force acting on a dust grain, as it is customary in all publications relating to the cometary dust removal. Strictly speaking, this is only physically reasonable if the back flux and corresponding inward momentum can be ignored. However, it is known through experiments (Gundlach et al. 2011) as well as from theory (Skorov et al. 2011, and references therein) that a porous media has a diffusion resistance that rapidly grows with increasing thickness. For example, only about $10 \%$ of the sublimating molecules enter the coma from a layer with dimensionless thickness of $20 R_{\mathrm{A}}$. The momentum associated with the remaining the molecules does not create a lifting force. To correctly evaluate the condition for dust removal, we therefore have to compare a tensile strength with the pressure gradient inside the porous layer. The pressure gradient and not the pressure itself produces the lifting force. To accurately model the distribution within the random porous layer, the modeling needs to be based on the kinetic theory of gases. However, in the first approximation, we can use the results of the permeability of the porous layer. To evaluate the distribution of pressure, it is enough to know the intensity of the upward gas flow as a function of the distance from the sublimating icy surface. Then a pressure change per one agglomerate sublayer can be extracted from the upward and inward gas fluxes.

Figure 4 shows the distribution of a pressure drop inside the porous media, normalized to the saturation pressure. The abscissa expresses the dimensionless distance from the sublimating icy surface, meaning that zero is at the ice-dust interface. The ordinate expresses $p\left((N+1) \times\left(2 R_{\mathrm{A}}\right)\right)-p\left(N \times\left(2 R_{\mathrm{A}}\right)\right) / p_{\text {sat }}$, that is, the normalized pressure drop per grain size. This calculation assumes that the gas temperature and its velocity distribution function are fixed inside the layer. Two different cases are shown that vary in their dependence on the assumption about the layer 

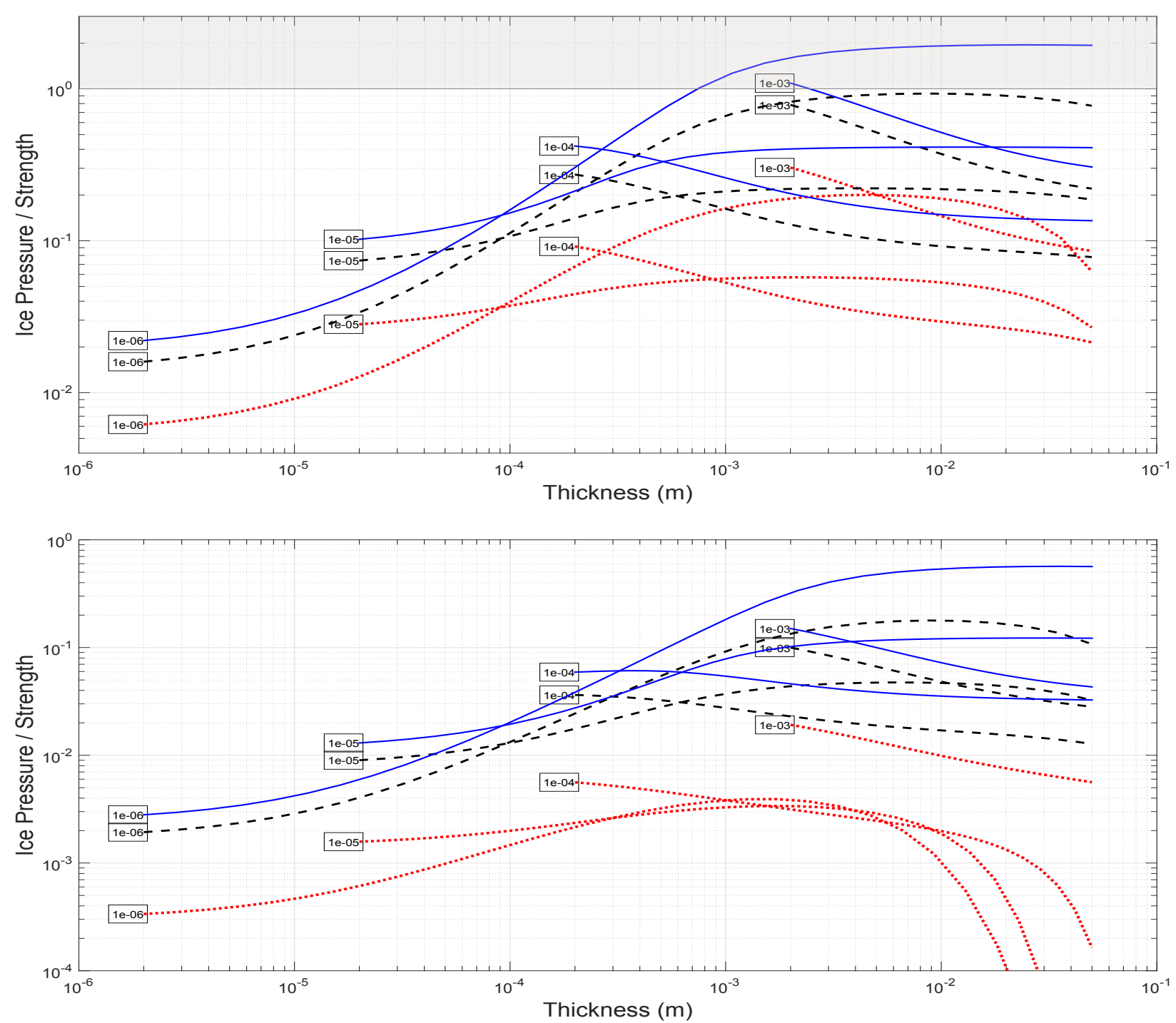

Fig. 3. Ratio of the vapor pressure above the sublimating ice to the tensile strength between constituting porous aggregates (radii are shown in the figure) as a function of the layer thickness (top panel for illumination corresponding to $R_{\mathrm{H}}=1.243 \mathrm{AU}$, bottom panel $R_{\mathrm{H}}=3.4 \mathrm{AU}$ ). Results are presented for water ice (dotted curves), carbon dioxide (dashed curves), and carbon monoxide (solid curves) ices.

permeability. The first case is for a modified Clausing function (Skorov et al. 2011), and the second case considers an experimental function evaluating a Knudsen diffusion through a mono-disperse random porous layer (Gundlach et al. 2011). The Clausing formula (dashed curves) predicts a more rapid decrease of the outflow intensity, and the dimensionless pressure drop is always smaller than the saturation vapor pressure. Similarly, using the experimental function for the layer permeability (solid curves), the pressure drop per agglomerate size is several times smaller than the saturation pressure. In addition, we note that on average the normalized pressure decrease goes down as the layer thickness increases. The apparent growth of a pressure near the surface after reaching the minimum (the right end of the curve) is a byproduct of the simplifying numerical treatment, an assumption that there is no back flux from outside. Under the real physical conditions, this flux might be from zero (for a collisionless flow) to about $20 \%$ (for the sublimation into vacuum from infinite plane). The relative peak near the icy surface (the left end of the curve) is also explained by the model assumption that sublimating molecules have a semi-Maxwellian velocity distribution. The pressure drop in the middle of the layer should therefore be considered as the most representative value. This brings us to an interesting conclusion: it is very unlikely that individual particles or fragments are removed from the porous hierarchic layer by the lifting force generated by sublimation of near-surface ice. This conclusion is valid for the types of ice we considered here $\left(\mathrm{H}_{2} \mathrm{O}, \mathrm{CO}_{2}, \mathrm{CO}\right)$ and for the agglomerate sizes we have tested. In the case of the smallest aggregates $(\sim 1 \mu \mathrm{m})$, a complete layer destruction (removal) is more physical than its internal fragmentation because the pressure change per aggregate (i.e., a resulting lifting force) is very small. This also means that if a dust cover is made of micron-sized aggregates, submillimeter particles can still be formed in the process of breaking or lifting the layer.

\section{Results and conclusion}

We investigated the role of dust cohesion and the effectivity of near-surface ice sublimation to lift dust particles ( $1 \mu \mathrm{m}$ to $1 \mathrm{~mm}$ ) from the nucleus surface. In the introductory overview we presented the historical development of this important topic, including the recent results of the Rosetta mission.

We presented results for a 1D model of the heat and mass transport into a two-layered medium, where a near-surface 


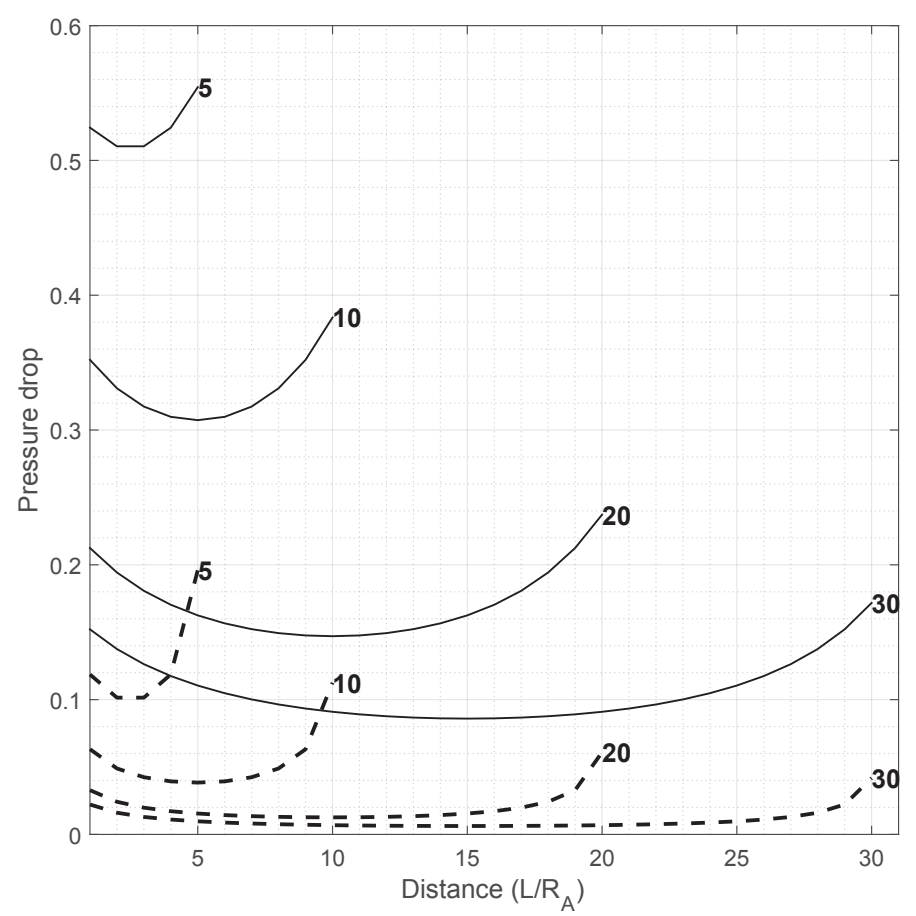

Fig. 4. Pressure drop per aggregate size inside a porous hierarchic dust layer as a function of the dimensionless distance from the sublimating ice surface. The layer thickness is denoted on the curves. See text for a precise definition of the pressure drop in Sect. 4.

sublimating ice-dust mixture is covered by an ice-free porous layer constructed from monodisperse porous agglomerates. The surface layer is irradiated by the Sun, and two illumination study cases were described that corresponded to different heliocentric distances and/or local irradiation conditions. Solid and radiative conductivity were both taken into account in the model. The layer permeability was evaluated from the theoretical model as well as from laboratory experiments. This work extends the models and results described in Skorov \& Blum (2012), Gundlach et al. (2015) by explicitly including the diffusivity resistance of the dust layer and consistent heat conductivity into the interior. As a result, the previous conclusions are updated, pointing even further into the direction that small $(<1 \mathrm{~mm})$ dust grains cannot be lifted at all from the nucleus by ice sublimation $\left(\mathrm{H}_{2} \mathrm{O}, \mathrm{CO}_{2}\right.$, or $\left.\mathrm{CO}\right)$.

At the first stage we investigated the dependence of vapor pressure above the icy boundary on model parameters: irradiation intensity, agglomerate size, and layer thickness, as done in previous works. The so-called cooking effect was accurately reproduced, such that the temperature of the icy interface was significantly increased when covered by a layer of fine agglomerates relative to the case of a bare ice surface (see Fig. 1). However, with increasing particle size, this effect rapidly weakens and disappears for the dust layer formed by coarse grains $\left(R_{\mathrm{A}} \sim 100 \mu \mathrm{m}\right)$. Given the dependence of vapor pressure on temperature, the pressure increase above the sublimating icy layer is also observed in only a limited range of agglomerate sizes and layer thicknesses. For example, at perihelion distance for grains $\left(R_{\mathrm{A}}<30 \mu \mathrm{m}\right)$, the water vapor pressure below a porous dust layer is about 30 times higher than the saturation pressure of open irradiated dark ice.

In addition, two scenarios for the behavior of vapor pressure versus layer thickness are observed. For the coarse grains $\left(R_{\mathrm{A}} \geq\right.$ $100 \mu \mathrm{m})$ the pressure below the dust layer is a monotonically decreasing function of its thickness. In that case, the conditions for dust lifting become weaker with the growth of the layer thickness. Therefore, once such a layer is formed, it remains stable and cannot be removed easily. When the layer is formed from fine grains, the vapor pressure grows with increasing layer thickness until it reaches a maximum value at a thickness of a few millimeters. Then the pressure decreases again as the layer grows because of the reduced heat flux at the ice boundary. The model simulations also showed that for $\mathrm{CO}_{2}$ and especially for $\mathrm{CO}$ ice, the pressure non-monotonicity is much weaker and does not develop the clear maximum in temperature/pressure curves. This is a result of the lower latent heat of $\mathrm{CO}$ sublimation (and valid for any super-volatiles). The small temperature difference between ice layer and core temperature renders the heat flux to the interior negligible, which means that more than enough energy remains to continued sublimation despite the growing layer thickness.

The feasibility of removing dust by the lifting force of a sublimating gas is summarized in Fig. 3, the ratio of a vapor pressure to the tensile strength. It should be understood that these calculations produce the upper limit for this process. The figure shows that the pressure of sublimating water ice is three orders of magnitude too low to overcome the tensile strength. Furthermore, even for the artificial case of super-volatile ices $\left(\mathrm{CO}_{2}\right.$ and $\left.\mathrm{CO}\right)$ that exist near the surface this process is inefficient in removing the dust from the surface. Only the micrometer-sized agglomerates may be lifted by the $\mathrm{CO}$ gas. However, at large heliocentric distance $\left(R_{\mathrm{H}}=3.4 \mathrm{AU}\right)$, the tensile strength between dust agglomerates always exceeds the vapor pressure.

We also focused on another important point: the real lifting force inside a porous layer is determined by the pressure drop, not by saturation vapor pressure; to break a bound between grains, a pressure gradient is needed. In general this characteristic is a complex function of the distance from the surface. However, its expected value can be easily evaluated from the diffusion resistance of the layer. The normalized pressure drop decreases for increasing layer thickness, and in all cases, this typical value is several times lower than the corresponding saturation pressure. As a result, the physically reasonable condition of dust removal is significantly less likely than the condition that follows from the simple comparison of saturation pressure and tensile strength.

Finally, we would like to make additional remark concerning the application of the typical thermophysical models to porous media. In this work the maximum size of dust agglomerates that was considered was $\sim 1 \mathrm{~mm}$. This restriction immediately follows from using a Fourier form of the heat transfer equation, which is only applicable when the grain sizes are smaller than the typical skin depth of a heat wave. Because of the low thermal inertia of 67P (Schloerb et al. 2015), an accurate simulation of a porous layer made of larger aggregates $\left(R_{\mathrm{A}} \geq 1 \mathrm{~cm}\right)$ is only possible with a discrete media approach.

In summary, within the framework of the widely used thermophysical models that account for the relevant physics, dust removal by a gas-lifting force is not possible. Even a super-volatile ice (i.e., CO) situated close to the surface (strongly unphysical assumption) cannot remove dust grains even for perihelion-like flux conditions, except for a very narrow range of conditions (Fig. 3). A way out of the impasse requires focusing on a revised common model assumption listed at the beginning of this section. It is evident that models in which the cohesion is ignored do not provide a consistent physical picture of the real dust activity on comets. 
Acknowledgements. We acknowledge the entire European Space Agency (ESA) Rosetta team and thank them for their contribution, without which this work could not have been done. Rosetta is an ESA mission with contributions from its member states and NASA. We also thank the International Space Science Institute (Bern) for giving us the opportunity to discuss this work with the international team on "Physical Properties of Cometary Nuclei Assessed from the Development of 67P CG's Activity".

\section{References}

Bentley, M. S., Schmied, R., Mannel, T., et al. 2016, Nature, 537, 73

Brisset, J., Heißelmann, D., Kothe, S., Weidling, R., \& Blum, J. 2016, A\&A, 593, A3

Chapman, C. R. 2004, Earth Plan. Sci. Lett., 222,

Combi, M. R. 1994, ApJ, 108, 304

Davidsson, B. J. R., Gutiérrez, P. J., Sierks, H., et al. 2015, A\&A, 583, A16

Della Corte, V., Rotundi, A., Fulle, M., et al. 2015, A\&A, 583, A13

Fanale, F. P., \& Salvail, J. R. 1984, Icarus, 60, 476

Groussin, B., Jorda, L., Auger, A.-T., et al. 2015, A\&A, 583, A32

Gundlach, B., \& Blum, J. 2012, Icarus, 219, 618

Gundlach, B., Skorov, Y. V., \& Blum, J. 2011, Icarus, 213, 710

Gundlach, B., Blum, J., Keller, H. U., \& Skorov, Y. V. 2015, A\&A, 583, A12

Hilchenbach, M., Kissel, J., Langevin, Y., et al. 2016, ApJ, 816, L32

Huebner, W. F., Benkhoff, J., Capria, M.-T., et al., 2006, Heat and Gas Diffusion in Comet Nuclei (Noordwijk, The Netherlands: ESA Publications Division)

Kalinichenko, A. I., Vasilyev, S. V., \& Vasilyev, V. P. 1996, Earth, Moon, and Planets, 74,17

Keller, H. U., Marconi, M. L., \& Thomas, N. 1990, A\&A, 227, L1
Keller, H. U., Mottola, S., Davidsson, B., et al. 2015, A\&A, 583, A34 Kramer, T., \& Noack, M. 2016, ApJ, 823, L11

Kuehrt, E., \& Keller, H. U. 1994, Icarus, 109, 121

Langevin, Y., Hilchenbach, M., Ligier, N., et al. 2016, Icarus, 271, 76

Lin, Z.-Y., Lai, I.-L., Su, C.-C., et al. 2016, A\&A, 588, L3

Marschall, R., Su, C. C., Liao, Y., et al. 2016, A\&A, 589, A90

Mavko, G., Mukerji, T., \& Dvorkin, J. 2009, The Rock Physics Handbook: Tools for Seismic Analysis of Porous Media, 2nd edn. (Cambridge: Cambridge University Press)

Mendis, D. A., Hill, J. R., Houpis, H. L. F., \& Whipple, E. C. 1981, ApJ, 249, 787

Moreno, F., Snodgrass, C., Hainaut, O., et al. 2016, A\&A, 587, A155

Nordheim, T. A., Jones, G. H., Halekas, J. S., Roussos, E., \& Coates, A. J. 2015, Planet. Space Sci., 119, 24

Orosei, R., Capaccioni, F., Capria, M. T., et al. 1995, A\&A, 301, 613

Rickman, H., Fernandez, J. A., \& Gustafson, B. S. 1990, A\&A, 237, 524

Rotundi, A., Sierks, H., Della Corte, V., et al. 2015, Science, 347

Schloerb, F. P., Keihm, S., von Allmen, P., et al. 2015, A\&A, 583, A29

Schulz, R., Hilchenbach, M., Langevin, Y., et al. 2015, Nature, 518, 216

Skorov, Y., \& Blum, J. 2012, Icarus, 221, 1

Skorov, Y. K., Rezac, L., Hartogh, P., Bazilevsky, A. T., \& Keller, H. U. 2016, A\&A, 999, 76

Skorov, Y. V., Kömle, N. I., Markiewicz, W. J., \& Keller, H. U. 1999, Icarus, 140,173

Skorov, Y. V., van Lieshout, R., Blum, J., \& Keller, H. U. 2011, Icarus, 212, 867

Spohn, T., Knollenberg, J., Ball, A. J., et al. 2015, Science, 349, 6247

Tubiana, C., Snodgrass, C., Bertini, I., et al. 2015, A\&A, 573, A62

Weidling, R., Güttler, C., \& Blum, J. 2012, Icarus, 218, 688

Whipple, F. L. 1950, ApJ, 111, 375 